\title{
Inhibition of IL-6/STAT3 axis and targeting Axl and Tyro3 receptor tyrosine kinases by apigenin circumvent taxol resistance in ovarian cancer cells
}

\author{
YOUNG-AH SUH ${ }^{1}$, SE-YOUNG JO ${ }^{1}$, HWA-YOUNG LEE ${ }^{2}$ and CHUHEE LEE ${ }^{2}$ \\ ${ }^{1}$ Institute for Innovative Cancer Research, Asan Medical Center, University of Ulsan College of Medicine, \\ Seoul 138-736; ${ }^{2}$ Department of Biochemistry and Molecular Biology, School of Medicine, \\ Yeungnam University, Daegu 705-717, Republic of Korea
}

Received October 1, 2014; Accepted November 26, 2014

DOI: $10.3892 / \mathrm{ijo} .2014 .2808$

\begin{abstract}
Ovarian cancer is the number one cause of death from gynaecological malignancy. Platinum-based and taxolbased chemotherapy has been used as a standard therapy, but intrinsic and acquired resistance to chemotherapy is a major obstacle to treat the disease. In the present study, we found that in the chemoresistant ovarian cancer SKOV3/TR cells, interleukin-6 (IL-6), IL-6 receptor and signal transducers and activators of transcription 3 (STAT3) expression as well as STAT3 phosphorylation were upregulated compared to those in parental cells. Silencing of IL-6 using IL-6 siRNA was found to suppress IL-6 production, STAT3 and phosphoSTAT3 levels, which eventually reduced proliferation and clonogenicity of taxol-resistant SKOV3/TR cells. In addition, stattic, a STAT3 inhibitor, was found to result in decrease of cell viability and clonogenicity of these cells, indicating that the elevated IL-6 and STAT3, phosphoSTAT3 levels are associated with the development of taxol resistance. Next, we found anti-proliferative effect of apigenin on both SKOV3 and SKOV3/TR cells. RT-PCR and western blot results showed that apigenin significantly reduced the expression of Axl and
\end{abstract}

Correspondence to: Dr Young-Ah Suh, Institute for Innovative Cancer Research, Asan Medical Center, University of Ulsan College of Medicine, Seoul 138-736, Republic of Korea

E-mail: ysuh925@gmail.com

Dr Chuhee Lee, Department of Biochemistry and Molecular Biology, School of Medicine, Yeungnam University, 317-1 Daemyung-5 Dong, Daegu 705-717, Republic of Korea

E-mail: chlee2@ynu.ac.kr

Abbreviations: Bcl-xl, B-cell lymphoma-extra large; ELISA, enzymelinked immunosorbent assay; GAS 6, growth arrest-specific 6; IL-6, interleukin-6; RTK, receptor tyrosine kinase; STAT, signal transducers and activators of transcription

Key words: Axl, apigenin, IL-6, ovarian cancer, STAT3, taxol resistance, Tyro3
Tyro3 receptor tyrosine kinases (RTKs) at mRNA and protein level, which account for its cytotoxic activity. We further found that apigenin decreased Akt phosphorylation and the level of B-cell lymphoma-extra large (Bcl-xl or BCL2-like 1 isoform 1), an inhibitor of apoptosis. On the contrary to these results, apigenin had no effect on IL-6 production, STAT3 and phosphoSTAT3 protein levels, suggesting that apigenin exerts its anti-proliferative activity via downregulation of Axl and Tyro3 expression, Akt phosphorylation and Bcl-xl expression, but not modulation of IL-6/STAT3 axis. Taken together, our data suggest that inhibition of IL-6/STAT3 signaling pathway and downregulation of Axl and Tyro3 RTKs expression might be a therapeutic strategy to overcome taxol resistance in ovarian cancer cells.

\section{Introduction}

Ovarian cancer is in the fifth place in respect of cancer-related death in woman (1). Worldwide the annual number of new cases and deaths of ovarian cancer is estimated to be around 0.22 and 0.14 million, respectively (2). Combination of surgery and chemotherapy has been used as a standard therapy for the treatment of ovarian cancer patients, but overall 5 -year survival of the patients with stage III and IV still remains at only 20 to $40 \%$. Such poor prognosis of advanced stage ovarian cancer is accounted for by the intrinsic and acquired chemoresistance, since $30 \%$ of patients with advanced stages have been reported not to respond to the first-line chemotherapy, paclitaxel and cisplatin/carboplatin, and $\sim 80 \%$ of the initial responders eventually relapse and develop chemoresistance (3). However, the underlying molecular mechanisms of chemoresistance in ovarian cancer are not fully understood.

The signal transducers and activators of transcription (STAT) family proteins have been reported to be fairly upregulated and constitutively activated in many tumors (4) and known to be resulted from the upregulation of upstream signaling molecules such as interleukin-6 (IL-6) (5). Of note, in half of ovarian cancers, constitutive activation of STAT3 has been observed and considered to play an important role for growth, cell cycle progression and invasion of these cancer cells (4). Therefore, targeting IL-6/STAT3 signaling 
axis via inhibition of the IL-6-IL-6R interaction or abrogation of STAT3 activation might be a clinically potential therapy to treat cancers.

Among more than 90 protein tyrosine kinases identified in the human genome, there are 58 receptor tyrosine kinases (RTK), which are classified into 20 families (6). One of the subfamily of RTK is the TAM family including three RTKs; Tyro3 (also called Sky), Axl (also called Ark and Ufo) and Mer (7). All the TAM RTKs have structural similarities, two immunoglobin-like domains and two fibronectin type III repeats in extracellular region and cytosolic kinase domain (8), and are recognized by growth arrest-specific 6 (Gas 6) and protein $\mathrm{S}$ in common, which are vitamin K-dependent proteins. In normal cells, intracellular signaling via TAM RTKs has been reported to be responsible for a various cellular functions such as survival, proliferation, blockage of apoptosis, adhesion, morphology and motility $(9,10)$. However, in cancer cells, it plays a critical role in the initiation as well as progression of cancers, since Axl, Mer and/or Gas 6 have been demonstrated to be overexpressed in a variety of cancer cell lines and patient samples including breast (11), colon (12), gastric (13), leukemias (14), melanoma (15), multiple myeloma (16), ovarian (17) and prostate cancer (18). Therefore, expression level of TAM RTKs as well as their ligands and their changes seem to be good prognostic marker and targeting these RTKs and their signaling pathways might be a feasible strategy for the successful treatment of many cancers.

Apigenin (4',5,7,-tirhydoxyflavone), a dietary flavone, is found in many fruits, vegetables and seasonings (19-22) as a dimer. Anti-proliferative and anti-angiogenic property of apigenin has been demonstrated in various cancers including breast (23), cervical (23), lung (24), colon (25), hematologic (26), ovarian (27) and prostate cancer (28). Based on these unique effects of apigenin on various cancers along with its low intrinsic toxicity, apigenin has received great attention as a therapeutic as well as a chemopreventive agent.

In the present study, we demonstrated that inhibition of IL-6/STAT3 axis and targeting Axl and Tyro3 RTKs resulted in the reduced proliferation of both parental SKOV3 cells and taxol-resistant SKOV3/TR cells, suggesting a therapeutic potential of these approaches to improve the overall outcome of patients with chemoresistant ovarian cancer.

\section{Materials and methods}

Reagents and antibodies. Apigenin was from Sigma-Aldrich (St. Louis, MO, USA). Primers for Axl, IL-6, IL-6 receptor, Mer, STAT3, Tyro3 and GAPDH were synthesized by a domestic company, Bioneer Corp. (Daejoun, Korea). TRI reagent was from Solgent (Daejoun, Korea). AmpliTaq DNA polymerase was obtained from Roche Inc. (Indianapolis, IN, USA). Enzyme-linked immunosorbent assay (ELISA) kit for interleukin 6 (IL-6) was obtained from R\&D Systems (Minneapolis, MN, USA). For immunoblotting, specific antibodies against Axl, STAT3, phosphoSTAT3, Tyro3 and GAPDH and secondary antibodies were obtained from Santa Cruz Biotechnology Inc. (Dallas, TX, USA).

Cell culture. SKOV3 cells were purchased from the American Type Culture Collection (ATCC, Manassas, VA, USA). The cells were grown in RPMI-1640 (Gibco-BRL, Grand Island, NY, USA) containing 10\% FBS, 2 mM L-glutamine, $10 \mathrm{U} / \mathrm{ml}$ penicillin and $10 \mathrm{~g} / \mathrm{ml}$ streptomycin at $37^{\circ} \mathrm{C}$ in $5 \% \mathrm{CO}_{2}$ in a water-saturated atmosphere. The taxol-resistant SKOV3/TR cells were established by stepwise exposure of the parental SKOV3 cells to escalating concentrations of taxol, ranging from 1.5 to $24 \mathrm{nM}$ for more than 6 months.

$R T$-PCR. Cells $\left(3 \times 10^{5}\right)$ were seeded in $60-\mathrm{mm}$ culture dish and grown overnight at $37^{\circ} \mathrm{C}$ and then treated with the indicated concentrations of apigenin for the $24 \mathrm{~h}$. Total RNA was extracted using TRI reagent and subjected to the cDNA synthesis and PCR. The specific primers were as follows: Axl, sense 5'-AACCTTCAACTCCTGCCTTCTCG-3' and antisense 5'-CAGCTTCTCCTTCAGCTCTTCAC-3'; Tyro3, sense 5'-GTGTGTGGCTGACTTCGGAC-3' and antisense 5'-CAC GTCCTCCATACACTCCG-3'; IL-6, sense 5'-ATGAACTCCT TCTCCACAAGCG-3' and antisense 5'-GAAGAGCCCTCA GGCTGGACT-3'; IL-6 receptor, sense 5'-CATTGCCATTGT TCTGAGGTTC-3' and antisense 5'-AGTAGTCTGTATTG CTGATGTC-3'; STAT3, sense 5'-TTCTCCTTCTGGGTCTG GCT-3' and antisense 5'-CCACCCAAGTGAAAGTGACG-3'; GAPDH, sense 5'-GGAGCCAAAAGGGTCATCAT-3' and antisense 5'-GTGATGGCATGGACTGTGGT-3'.

Western blot analysis. Cells were treated with the indicated concentration of apigenin or stattic for $24 \mathrm{~h}$. Total cell lysates were prepared from those cells using lysis buffer [1\% Triton X-100, $50 \mathrm{mM}$ Tris ( $\mathrm{pH} 8.0$ ), $150 \mathrm{mM} \mathrm{NaCl}, 1 \mathrm{mM}$ PMSF, $1 \mathrm{mM} \mathrm{Na} \mathrm{VO}_{4}$, and protease inhibitor cocktail]. Protein concentrations were determined using Bio-Rad protein assays. Proteins from cell lysates $(20-40 \mu \mathrm{g})$ were separated on $12 \%$ SDS-PAGE, and electrotransferred to nitrocellulose membranes. Membranes were blocked for $30 \mathrm{~min}$ at room temperature in Tris-buffered saline-0.05\% Tween-20 (TTBS) containing $5 \%$ non-fat dry milk, and then incubated with TTBS containing a primary antibody for $4 \mathrm{~h}$ at room temperature. After 3 x 10-min washes in TTBS, membranes were incubated with peroxidase-conjugated secondary antibody for $1 \mathrm{~h}$. Following 3 additional 10-min washes with TTBS, protein bands of interest were visualized using an enhanced chemiluminescence detection system (Amersham).

siRNA transfection. RNA interference silencing was performed to inhibit IL-6 production. SKVO3/TR cells $\left(1 \times 10^{6}\right)$ were seeded in 100-mm culture dish and grown overnight and then transfected with 50 nM siRNA against IL-6 (sc-39627; Santa Cruz Biotechnology, Dallas, TX, USA), or control siRNA (sc-37007; Santa Cruz Biotechnology). At 48 h posttransfection, cells were harvested and the number of viable cells were counted and IL-6 level in conditioned media were determined by ELISA. STAT3 and phosphorylated STAT3 protein levels were determined by western blot analysis using whole cell lysates.

Clonogenic assay. Cells were seeded in 35-mm culture dishes ( $2 \times 10^{3}$ cells/dish) and allowed to grow for 7-10 days in the presence of and/or absence of apigenin or stattic to form colonies. Colonies of $>50$ cells were visualized by crystal violet (in $60 \%$ methanol; Junsei Chemical Co., Ltd., Tokyo Japan) staining 

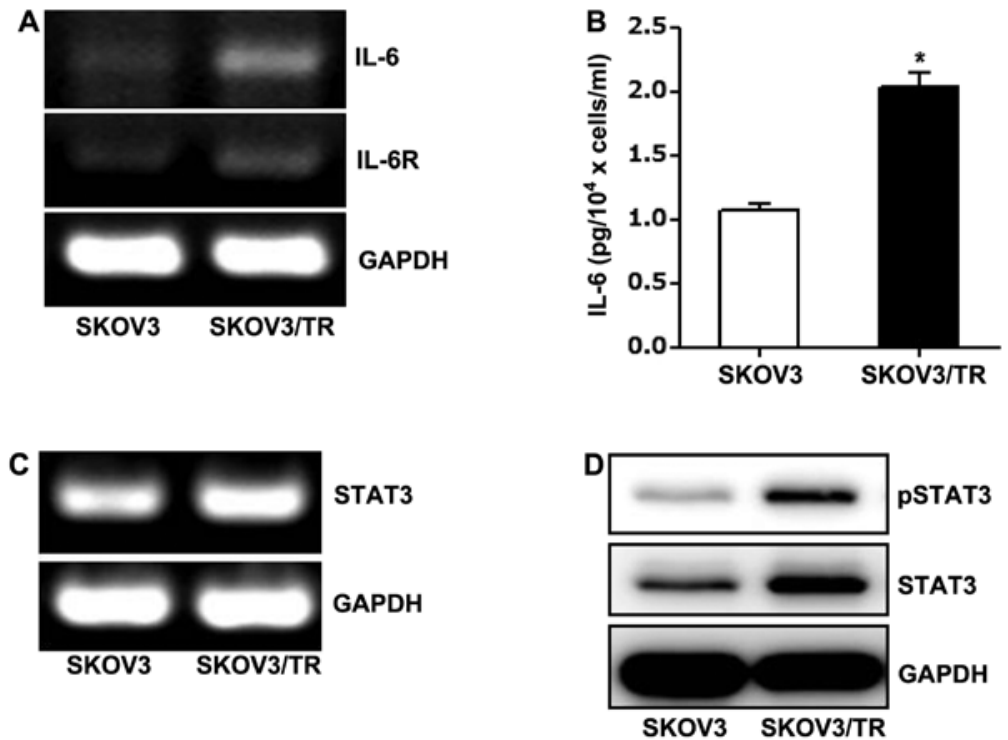

Figure 1. The levels of IL-6, IL-6 receptor, STAT3 and phosphorylated STAT3 were elevated in taxol-resistant ovarian cancer cells. (A and C) Total RNAs from SKOV3 and SKOV3/TR cells were isolated and used for RT-PCR to analyze $I L-6, I L-6$ and STAT3 mRNA expression. As an internal control, GAPDH mRNA was also amplified by RT-PCR. The data are representative of three independent experiments. (B) Culture media of both SKOV3 and SKOV3/TR cells were harvested and used for IL-6 ELISA. To assess IL-6 production per cell, the total amount of IL-6 was normalized by the number of viable cells. Results are from three independent experiments. Data are expressed as the mean \pm SD of triplicate samples. The asterisks indicate the significant difference compared to the control value ("P<0.05 SKOV3 vs. SKOV3/TR). (D) Western blot analysis was also conducted to determine the levels of STAT3 protein and its phosphorylated form in SKOV3 and SKOV3/TR cells. GAPDH was used as a loading control. The data are representative of three independent experiments.

and images were taken by RAS 3000 image analysis system (Fuji Film, Tokyo, Japan).

Cell viability assay. The viability of cells was measured using Cell Counting Kit-8 assay kit (Dojindo Laboratories,

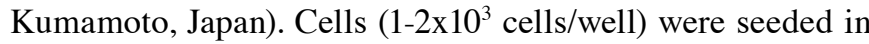
96-well plates and grown overnight at $37^{\circ} \mathrm{C}$ and then treated with the indicated concentrations of stattic for the $24 \mathrm{~h}$. At the end of the treatment, $10 \mu \mathrm{l}$ of CCK- 8 solution was added and further incubated for $4 \mathrm{~h}$. The absorbance at $450 \mathrm{~nm}$ was measured using a microplate reader (Model 680 microplate reader; Bio-Rad Laboratories). Values are the mean \pm SD for triplicate wells and normalized to that of control group to determine the $\%$ of viability.

ELISA. The level of IL-6 in culture media was measured using ELISA kit from R\&D Systems according to the manufacturer's protocol. Cells were transfected with siRNAs, siCtrl and silL-6 or treated with apigenin for $24 \mathrm{~h}$. Conditioned media were harvested and assayed for IL-6. The data are representative of at least three independent experiments.

Statistical analysis. Data are expressed as the mean \pm SD of triplicate samples or at least three independent experiments. For statistical significance, Student's t-test was used with a threshold of P-values which is $<0.05$.

\section{Results}

IL-6, STAT3 and phosphorylated STAT3 levels are elevated in taxol-resistant ovarian cancer cells. To understand the molecular mechanisms underlying taxol resistance in ovarian cancer cells, we established a taxol-resistant subline, SKOV3/TR cells, by long-term and stepwise exposure of taxol to parental SKOV3 cells. Since elevated production of interleukin-6 (IL-6) and IL-6-mediated activation of signal transducers and the activators of transcription 3 (STAT3) have been reported to lead to chemoresistance to several chemotherapeutic drugs in various cancers (29), we examined IL-6, IL-6 receptor, STAT3, and phosphorylated STAT3 levels in both SKOV3 and SKOV3/TR cells. RT-PCR result showed that IL-6 and IL-6 receptor mRNA levels in SKOV3/TR cells were increased compared to those of SKOV3 cells, respectively (Fig. 1A). In addition, enzyme-linked immunosorbent assay (ELISA) result also showed that the level of IL-6 in culture media of SKOV3/TR cells was higher than that in culture media of parental cells, which is consistent with the transcriptional upregulation of IL-6 in SKOV3/TR cells (Fig. 1B).

Next, expression and phosphorylation status of STAT3 were examined. As shown in Fig. 1C, STAT3 mRNA level in SKOV3/TR cells was found to be increased compared to that in parental cells. Western blot results also showed that in SKOV3/TR cells, both STAT3 protein and phosphoSTAT3 level were significantly elevated (Fig. 1D), indicating the induction of STAT3 expression and its activation might be responsible for the development of resistance to chemotherapy.

Silencing of IL-6 and inhibition of STAT3 reduce proliferation of taxol-resistant cells. The biological relevance of the increase of IL-6 production, STAT3 protein level, and its phosphorylation status in SKOV3/TR cells was examined by silencing of IL-6 via siRNA and inhibition of STAT3 using stattic, a small molecule inhibitor of STAT3. SKOV3/TR cells were transfected with IL-6-specific siRNA, siIL-6 or control siRNA, siCtrl and assessed IL- 6 level in culture media by ELISA. As shown in Fig. 2A, silencing of IL-6 via silL-6 

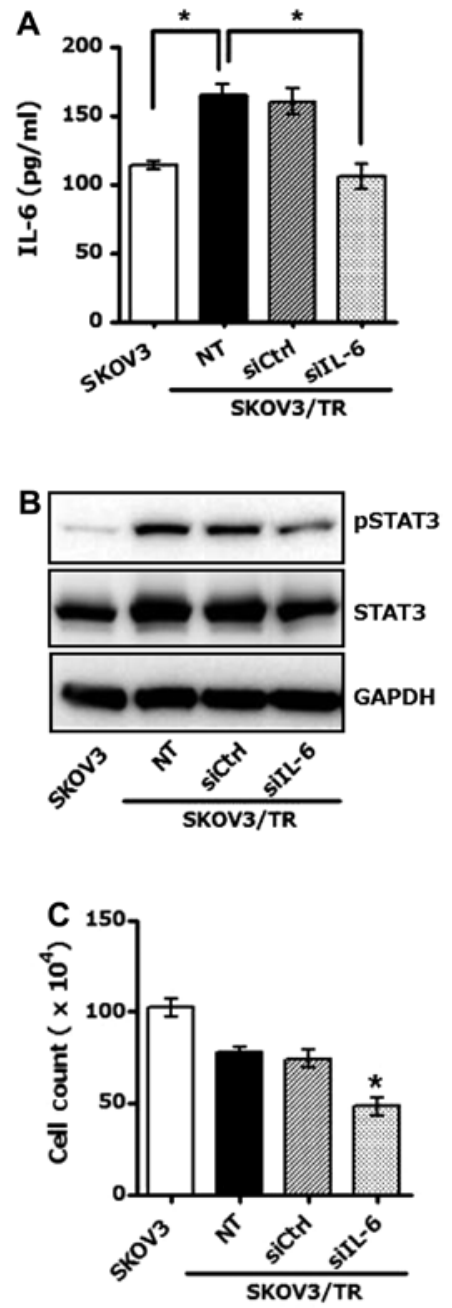

Figure 2. Silencing of IL-6 inhibits STAT3 expression and phosphorylation in taxol-resistant ovarian cancer cells, which eventually suppresses proliferation of these cells. (A) SKOV3/TR cells $\left(3 \times 10^{3}\right.$ cells) were transfected with IL-6-specific siRNA, siIL-6 and control siRNA, siCtrl, respectively. Cells were harvested $48 \mathrm{~h}$ after transfection and IL-6 protein level was determined by ELISA. The data are representative of three independent experiments. The asterisks indicate the significant difference compared to the control value ("P<0.05 SKOV3 vs. SKOV3/TR and SKOV3/TR vs. SKOV3/TR/siIL-6). (B) In parallel, the levels of STAT3 protein and its phosphorylated form were determined by western blot analysis. GAPDH was used as a loading control. The data are representative of three independent experiments. (C) To assess anti-proliferative effect of siIL-6, the number of viable cells was counted at $48 \mathrm{~h}$ post-transfection. Data are expressed as the mean \pm SD of three independent experiments. The asterisk indicates a significant difference compared to the control value ("P $<0.05$ SKOV3/TR vs. SKOV3/TR/siIL-6). The results are representative of at least three independent experiments.

in SKOV3/TR cells resulted in significant decrease of IL-6 production. Western blot results further showed that phosphorylation and expression of STAT3 was also reduced in SKOV3/TR cells transfected with silL-6 (Fig. 2B), confirming that STAT3 is a downstream effector of IL-6-mediated signaling pathway.

Next, we examined the effect of silL-6 on cell proliferation. As shown in Fig. 2C, viability of SKOV3/TR cells transfected with silL-6 was fairly reduced compared to that transfected with siCtrl.

The effect of stattic, a small molecule inhibitor of STAT3, on cell viability was also examined. We first found that both
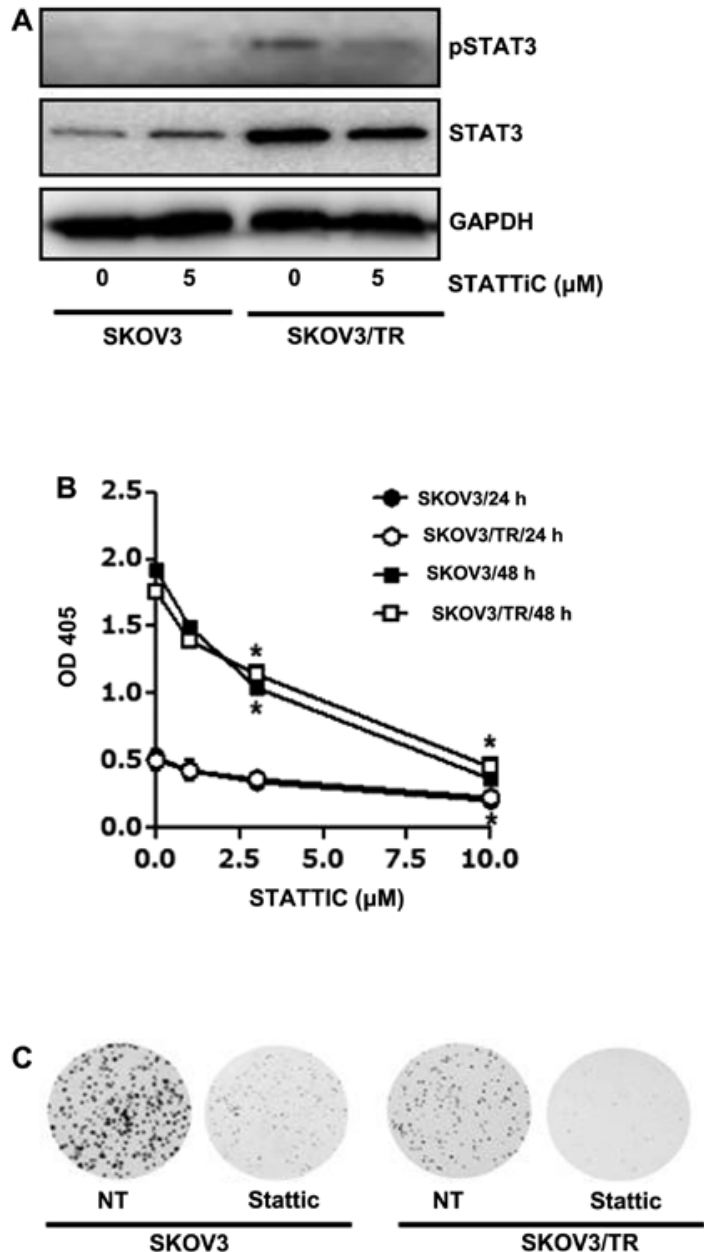

Figure 3. Stattic, a small molecule inhibitor, inhibits STAT3 phosphorylation and proliferation of taxol-resistant ovarian cancer cells. Cells $\left(3 \times 10^{3}\right.$ cells/dish) were seeded onto $100-\mathrm{mm}$ dishes, grown overnight and treated with the indicated concentrations of stattic for 24 or $48 \mathrm{~h}$. (A) After $24 \mathrm{~h}$ of treatment, cells were harvested. The levels of STAT3 protein and its phosphorylated form were determined by western blot analysis. GAPDH was used as a loading control. The data are representative of three independent experiments. (B) Cell viability was measured by CCK- 8 assay. Each error bar represents mean \pm SD of three independent experiments. The asterisk indicates a significant difference compared to the control value ( $\mathrm{P}<0.05$ vs. untreated group). (C) Each cell $\left(2 \times 10^{3}\right.$ cells/dish) was seeded onto $35-\mathrm{mm}$ dishes and treated with $5 \mu \mathrm{M}$ stattic. After $48 \mathrm{~h}$ of treatment, cells were washed with PBS and allowed to grow for 7 to 10 days. The colonies were visualized by crystal violet staining. The results are representative of three independent experiments.

expression and phosphorylation of STAT3 were decreased by stattic treatment in SKOV3/TR cells (Fig. 3A). Then, both SKOV3 and SKOV3/TR cells were treated with 1, 3 and $10 \mu \mathrm{M}$ stattic for $24 \mathrm{~h}$ and CCK assay results showed a dosedependent decrease of the cell viability (Fig. 3B). Consistent with the CCK assay results, we also found that clonogenicity of stattic-treated cells was considerably reduced (Fig. 3C), confirming its anti-proliferative activity. Taken together, these results demonstrated that upregulation of IL-6 and STAT3 expression as well as the increased phosphorylation of STAT3 play a critical role in proliferation of SKOV3/TR cells and are associated with taxol resistance of SKOV3/TR cells.

Apigenin suppresses proliferation of both parental and taxolresistant cells. Since we previously reported that apigenin 

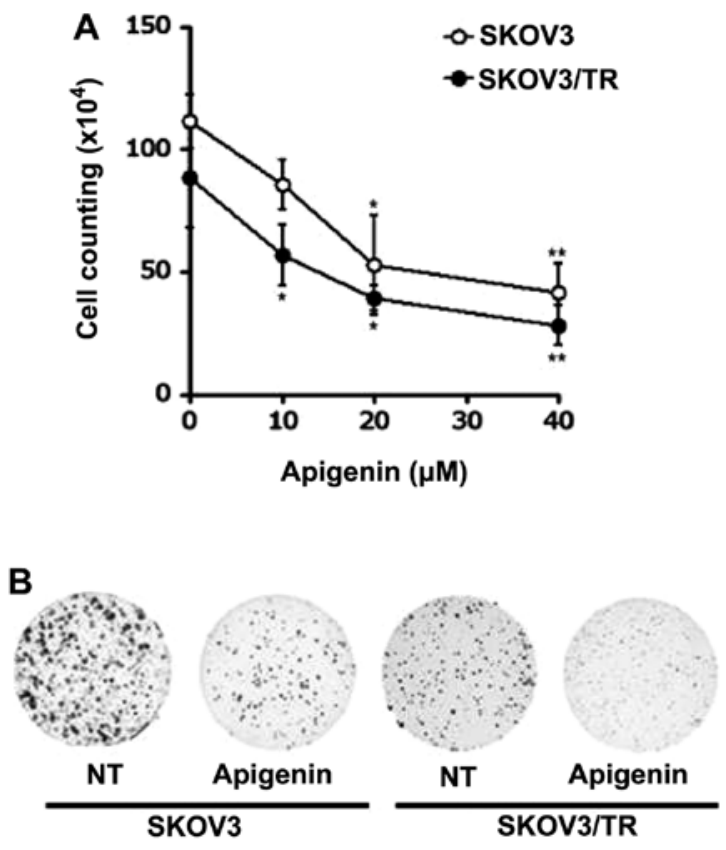

Figure 4. Apigenin inhibits proliferation of taxol-resistant ovarian cancer cells. (A) Cells $\left(3 \times 10^{3}\right.$ cells/dish) were seeded onto $100-\mathrm{mm}$ dishes, grown overnight and treated with the indicated concentrations of apigenin for $24 \mathrm{~h}$ and harvested. Trypan blue exclusion assay was conducted to measure cell viability. Data are presented as mean \pm SD of at least three independent experiments. The asterisks indicate a significant difference compared to the control value $\left({ }^{*} \mathrm{P}<0.05\right.$ vs. untreated group and ${ }^{* *} \mathrm{P}<0.001$ vs. untreated group). (B) Cells $\left(2 \times 10^{3}\right.$ cells/dish) were seeded onto $35-\mathrm{mm}$ dishes and allowed to grow in the absence or presence of $40 \mu \mathrm{M}$ apigenin for 7 to 10 days. The colonies were visualized by crystal violet staining. The data are representative of at least three independent experiments.

targets Axl receptor tyrosine kinase (RTK), one of TAM family members, which accounts for its anti-proliferative effects on non-small cell lung carcinoma (NSCLC) cell lines, we asked whether apigenin was cytotoxic in parental and taxol-resistant ovarian cancer cells, which might result from downregulation of TAM expression. As shown in Fig. 4A, apigenin treatment decreased the viability of both SKOV3 and SKOV3/TR cells in a dose-dependent manner. Of note, treatment with $40 \mu \mathrm{M}$ apigenin for $24 \mathrm{~h}$ showed only $41.5 \%$ (SKOV3), and 28\% (SKOV3/TR) survival of these cells, respectively (Fig. 4A), indicating a more profound anti-proliferative effect of apigenin on SKOV3/TR cells than parental SKOV3 cells. Colony-forming assay further demonstrated cytotoxic activity of apigenin on SKOV3 and SKOV3/TR cells. As shown in Fig. 4B, treatment of these cells with $40 \mu \mathrm{M}$ apigenin was found to reduce not only the number of colonies but also the size of each colony.

Anti-proliferative effect of apigenin is mediated by the dysregulation of TAM RTKs and downstream effectors, but not IL-6/STAT3 axis. Since TMA family of RTKs, Axl, Tyro3 and Mer is known to be involved in cell survival, growth and proliferation, we then examined the effect of apigenin on TAM RTKs expression. Especially, in SKOV3/TR cells, Axl expression was found to be slightly reduced, while Tyro3 expression was increased, compared to those in parental SKOV 3 cells, respectively. Both SKOV3 and SKOV3/TR cells were treated with $40 \mu \mathrm{M}$ apigenin for $24 \mathrm{~h}$ and then expres-
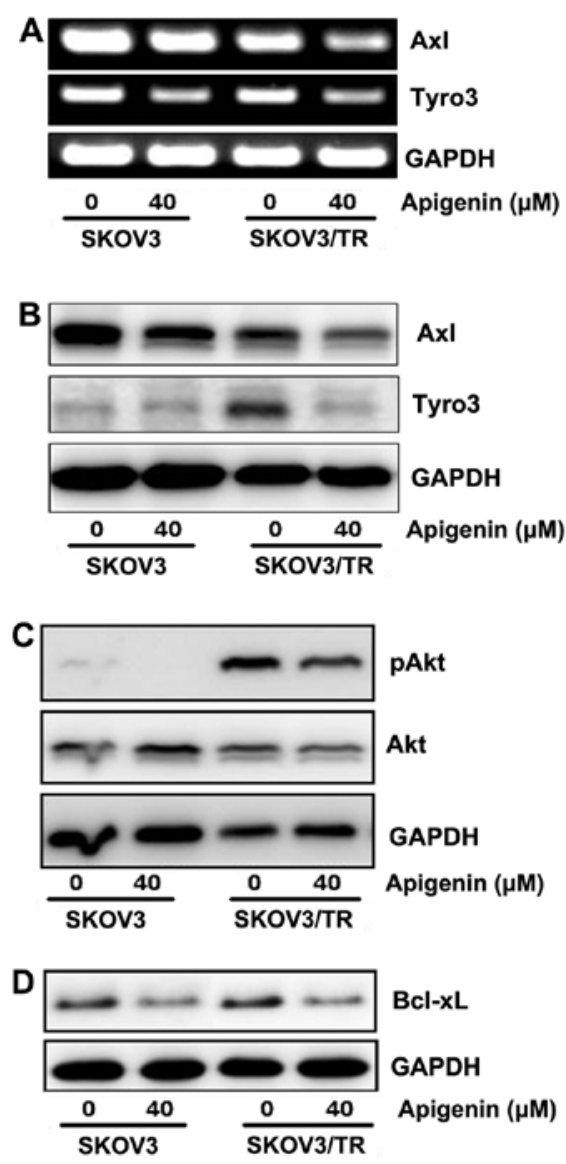

Figure 5. Apigenin suppresses the expression of TAM RTKs Axl and Tyro3 RTKs. Cells ( $3 \times 10^{3}$ cells/dish) were seeded onto $100-\mathrm{mm}$ dishes, grown overnight and treated $40 \mu \mathrm{M}$ apigenin for $24 \mathrm{~h}$ and harvested. (A) For RT-PCR, total RNAs from the cells were isolated and used for analysis of $A x l$ and Tyro3 mRNA expression. The data are representative of three independent experiments. As an internal control, GAPDH mRNA was also amplified. (B-D) The levels of Axl, Tyro 3, Akt, phosphorylated Akt and Bcl-xl protein were assessed by western blot analysis to determine the effect of apigenin on their expression. GAPDH was used as a loading control. The result are representative of at least three independent experiments.

sion of Axl and Tyro3 was examined at mRNA and protein level. RT-PCR results showed that apigenin treatment led to significant reduction of Axl and Tyro3 mRNA level in both parental and taxol-resistant cells (Fig. 5A). Downregulation of Axl and Tyro3 expression in apigenin-treated cells was further confirmed by western blot analysis. As shown in Fig. 5B, the protein levels of Axl and Tyro3 were decreased by apigenin treatment, which is consistent with RT-PCR results.

We next examined several downstream effectors which might be affected after apigenin-mediated inhibition of Axl and Tyro3 expression and subsequent reduction of cell proliferation. Western blot results showed that apigenin treatment decreased the level of phosphorylated Akt which transduces a strong signal for cell cycle progression and is fairly increased in SKOV3/TR cells (Fig. 5C). In addition, apigenin was also found to reduce the level of B-cell lymphoma-extra large (Bcl-xl, or BCL2-like 1 isoform 1) which is regulated by Akt and inhibits apoptosis (Fig. 5D). These data demonstrate that apigenin causes not only reduction of Axl and Tyro3 expression but also the decrease of Akt phosphorylation and Bcl-xl expression. 

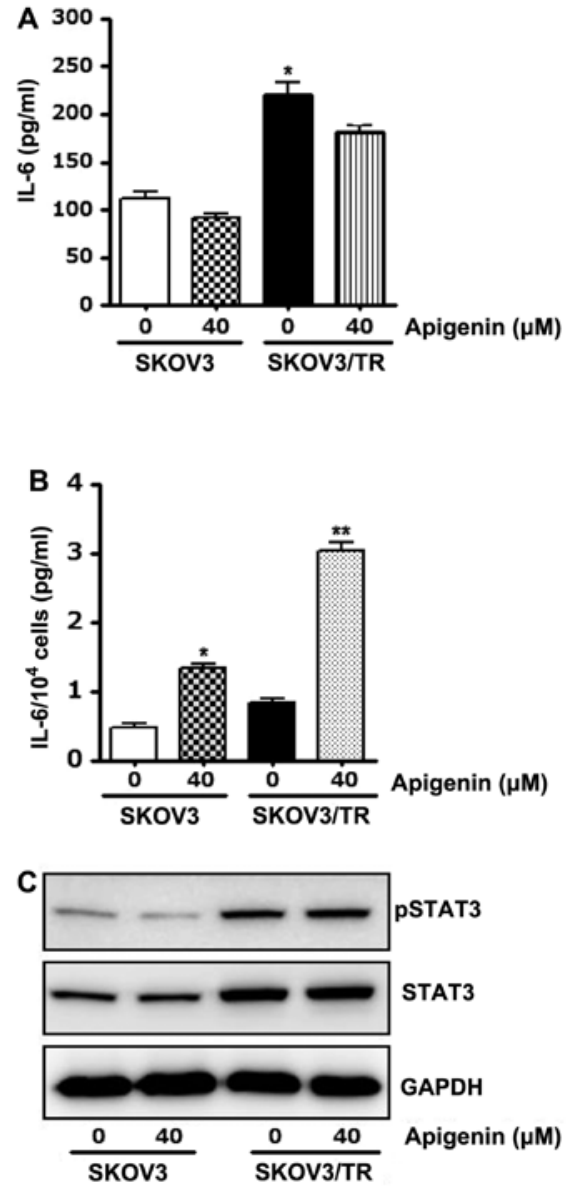

Figure 6. IL-6/STAT3 axis is not affected by apigenin. Cells $\left(3 \times 10^{3}\right.$ cells/dish $)$ were seeded onto $100 \mathrm{~mm}$ dishes, grown overnight and treated $40 \mu \mathrm{M}$ apigenin for $24 \mathrm{~h}$. (A) Culture media were harvested and used for IL-6 ELISA. Data are expressed as the mean \pm SD of triplicate samples. The asterisks indicate a significant difference compared to the control value ( ${ }^{*} \mathrm{P}<0.05$ vs. untreated). The results are representative of at least three independent experiments. (B) To assess IL-6 production per cell, the total amount of IL-6 was normalized by the number of viable cells. Data are expressed as the mean \pm SD of triplicate samples. The asterisks indicate a significant difference compared to the control value ( ${ }^{*} \mathrm{P}<0.05$ vs. untreated SKOV3 cells and ${ }^{* *} \mathrm{P}<0.001$ vs. untreated SKOV3/TR cells). (C) STAT3 and phosphorylated STAT3 protein levels were assessed by western blot analysis to determine the effect of apigenin on their expression. GAPDH was used as a loading control. The results are representative of at least three independent experiments.

We further examined if apigenin affects IL-6/STAT3 axis, which is associated with cell viability. ELISA results showed that total amount of IL-6 in culture media was slightly decreased by apigenin treatment (Fig. 6A), whereas IL-6 production per cell was increased, especially in taxolresistant SKOV3/TR cells (Fig. 6B). We also found that apigenin treatment had no effect on STAT3 phosphorylation in these cells (Fig. 6C). Taken together, the results indicate that apigenin has no effect on IL-6 production and concomitant STAT3 phosphorylation and its anti-proliferative effect does not result from suppression of IL-6/STAT3 axis.

\section{Discussion}

Cisplatin- and taxol-based chemotherapy is still the first-line therapeutic choice for ovarian cancer. However, the intrinsic and acquired resistance to these drugs have major limitations leading to the failure of treatment (30-33). Therefore, it is urgent to elucidate characteristics and underlying molecular mechanisms of the resistance to improve final outcomes.

We found that in taxol-resistant SKOV3/TR cells, the levels of IL-6, IL-6 receptor, STAT3 and its phosphorylated form were significantly increased compared to those in parental SKOV3 cells. Moreover, intervention of this IL-6/STAT3 signaling via silencing of IL-6 and a STAT3 inhibitor, stattic, were found to exert anti-proliferative effect on SKOV3/TR cells. These results indicate that activation of IL-6/STAT3 axis resulted from the long-term exposure of cells to taxol and need a strategy or a compensation to survive under the pressure of taxol. Because of dual function of STAT3 as a downstream effector of IL-6 and a transcription factor to induce IL-6 expression, a positive feedback loop between STAT3 and IL-6 is established, which results in autocrine production of IL- 6 and constitutive activation of STAT3. Consistent with our data, the anti-apoptotic effect of IL-6 and the involvement in drug resistance have been reported in various cancers including myeloma (34), prostate (35) and breast cancers (36), which supports the idea that combination of IL-6/STAT3 pathway inhibitor with chemotherapeutic agents could be effective, in patients with acquired chemoresistance.

A clinical significance of TAM receptor tyrosine kinases (RTKs), Tyro3, Axl and Mer, as well as their ligands has been demonstrated. For example, in $48.3 \%$ of ovarian adenocarcinoma tissues, Axl protein level was elevated and reflected in disease stage and lymph node metastasis. In lung cancer cases, overexpression of Axl, Mer, and their ligands was also found in more than half of non-small cell lung cancer (NSCLC) cell lines $(37,38)$ and RNA interference or monoclonal antibodies against Axl have been reported to reduce NSCLC proliferation, metastasis and xenograft tumor growth (39). In accordance with these studies, we recently demonstrated that anti-proliferative effect on apigenin, a dietary phytochemical derived from various fruits and vegetables resulted from downregulation of Axl expression in NSCLC cells, suggesting that Axl is a novel target of apigenin. Since the initial report showed the inhibitory effect of apigenin on mutagenesis and tumor promotion (40), many follow-up studies further demonstrated its anti-oxidant, anti-inflammatory, anti-angiogenic and anti-proliferative activities. Based on the above evidence, apigenin has received considerable attention as a chemotherapeutic and chemopreventive agent. In the present study, apigenin was further found to suppress the expression of Axl and Tyro3, incurring decreased proliferation of both parental and taxol-resistant SKOV3 cancer cells. Of note, Tyro3 induction contrary to downregulation of Axl in SKOV3/TR cells seems to be a compensation or another strategy for survival, which resulted from long-term treatment of taxol. However, IL-6 and STAT3 expression and STAT3 phosphorylation were not affected by apigenin, IL-6 production per cell was increased, suggesting that IL-6/STAT3 signaling pathway is not involved in the anti-proliferative effect of apigenin.

In summary, our data demonstrated that silencing of IL-6 and STAT3 inhibition intervened IL-6/STAT3 signaling pathway and apigenin caused downregulation of expression 
in all TAM RTKs, which eventually restricted in proliferation of taxol-resistant ovarian cancer cells, suggesting that inhibition of IL-6/STAT3 axis and targeting TAM RTKs might be feasible approaches to overcome taxol resistance in ovarian cancer cells.

\section{Acknowledgements}

The present research was supported by the Basic Science Research Program through the National Research Foundation of Korea (NRF) funded by the Ministry of Education, Science and Technology (grant no. 2006-2005303 and NRF2014R1A1A2006192).

\section{References}

1. Siegel R, Ma J, Zou Z and Jemal A: Cancer statistics, 2014. CA Cancer J Clin 64: 9-29, 2014.

2. Cohen JG, White M, Cruz A and Farias-Eisner R: In 2014, can we do better than CA125 in the early detection of ovarian cancer? World J Biol Chem 5: 286-300, 2014.

3. McGuire WP, Hoskins WJ, Brady MF, et al: Cyclophosphamide and cisplatin compared with paclitaxel and cisplatin in patients with stage III and stage IV ovarian cancer. N Engl J Med 334 1-6, 1996.

4. Levy DE and Darnell JE Jr: Stats: transcriptional control and biological impact. Nat Rev Mol Cell Biol 3: 651-662, 2002.

5. Chang KT, Tsai CM, Chiou YC, Chiu CH, Jeng KS and Huang CY: IL-6 induces neuroendocrine dedifferentiation and cell proliferation in non-small cell lung cancer cells. Am J Physiol Lung Cell Mol Physiol 289: L446-L453, 2005.

6. Robinson DR, Wu YM and Lin SF: The protein tyrosine kinase family of the human genome. Oncogene 19: 5548-5557, 2000.

7. Ohashi K, Mizuno K, Kuma K, Miyata T and Nakamura T: Cloning of the cDNA for a novel receptor tyrosine kinase, Sky, predominantly expressed in brain. Oncogene 9: 699-705, 1994.

8. Sasaki T, Knyazev PG, Clout NJ, et al: Structural basis for Gas6-Axl signalling. EMBO J 25: 80-87, 2006.

9. Stitt TN, Conn G, Gore M, et al: The anticoagulation factor protein $\mathrm{S}$ and its relative, Gas6, are ligands for the Tyro 3/Axl family of receptor tyrosine kinases. Cell 80: 661-670, 1995.

10. Hafizi S and Dahlback B: Gas6 and protein S: Vitamin K-dependent ligands for the Axl receptor tyrosine kinase subfamily. FEBS J 273: 5231-5244, 2006.

11. Meric F, Lee WP, Sahin A, Zhang H, Kung HJ and Hung MC: Expression profile of tyrosine kinases in breast cancer. Clin Cancer Res 8: 361-367, 2002.

12. Craven RJ, Xu LH, Weiner TM, et al: Receptor tyrosine kinases expressed in metastatic colon cancer. Int J Cancer 60: 791-797, 1995.

13. Wu CW, Li AF, Chi CW, et al: Clinical significance of AXL kinase family in gastric cancer. Anticancer Res 22: 1071-1078, 2002.

14. Challier C, Uphoff CC, Janssen JW and Drexler HG: Differential expression of the ufo/axl oncogene in human leukemia-lymphoma cell lines. Leukemia 10: 781-787, 1996.

15. Gyorffy B and Lage H: A Web-based data warehouse on gene expression in human malignant melanoma. J Invest Dermatol 127: 394-399, 2007.

16. De Vos J, Couderc G, Tarte K, et al: Identifying intercellular signaling genes expressed in malignant plasma cells by using complementary DNA arrays. Blood 98: 771-780, 2001.

17. Macleod K, Mullen P, Sewell J, et al: Altered ErbB receptor signaling and gene expression in cisplatin-resistant ovarian cancer. Cancer Res 65: 6789-6800, 2005.

18. Sainaghi PP, Castello L, Bergamasco L, Galletti M, Bellosta P and Avanzi GC: Gas6 induces proliferation in prostate carcinoma cell lines expressing the Axl receptor. J Cell Physiol 204: 36-44, 2005 .
19. Birt DF, Hendrich S and Wang W: Dietary agents in cancer prevention: flavonoids and isoflavonoids. Pharmacol Ther 90: 157-177, 2001.

20. Surh YJ: Cancer chemoprevention with dietary phytochemicals. Nat Rev Cancer 3: 768-780, 2003.

21. Manach C, Scalbert A, Morand C, Remesy C and Jimenez L: Polyphenols: food sources and bioavailability. Am J Clin Nutr 79: 727-747, 2004

22. Yang CS, Landau JM, Huang MT and Newmark HL: Inhibition of carcinogenesis by dietary polyphenolic compounds. Annu Rev Nutr 21: 381-406, 2001.

23. Zheng PW, Chiang LC and Lin CC: Apigenin induced apoptosis through p53-dependent pathway in human cervical carcinoma cells. Life Sci 76: 1367-1379, 2005.

24. Lu HF, Chie YJ, Yang MS, et al: Apigenin induces apoptosis in human lung cancer $\mathrm{H} 460$ cells through caspase- and mitochondria-dependent pathways. Hum Exp Toxicol 30: 1053-1061, 2011.

25. Zhong Y, Krisanapun C, Lee SH, et al: Molecular targets of apigenin in colorectal cancer cells: involvement of p21, NAG-1 and p53. Eur J Cancer 46: 3365-3374, 2010.

26. Ruela-de-Sousa RR, Fuhler GM, Blom N, Ferreira CV, Aoyama H and Peppelenbosch MP: Cytotoxicity of apigenin on leukemia cell lines: implications for prevention and therapy. Cell Death Dis 1: e19, 2010.

27. Li ZD, Hu XW, Wang YT and Fang J: Apigenin inhibits proliferation of ovarian cancer A2780 cells through Id1. FEBS Lett 583: 1999-2003, 2009.

28. Mirzoeva S, Kim ND, Chiu K, Franzen CA, Bergan RC and Pelling JC: Inhibition of HIF-1 alpha and VEGF expression by the chemopreventive bioflavonoid apigenin is accompanied by Akt inhibition in human prostate carcinoma PC3-M cells. Mol Carcinog 47: 686-700, 2008.

29. Barre B, Vigneron A, Perkins N, Roninson IB, Gamelin E and Coqueret O: The STAT3 oncogene as a predictive marker of drug resistance. Trends Mol Med 13: 4-11, 2007.

30. McGuire WP III: Current status of taxane and platinum-based chemotherapy in ovarian cancer. J Clin Oncol 21 (Suppl 10): 133-135, 2003

31. Cannistra SA: Cancer of the ovary. N Engl J Med 351: 2519-2529, 2004.

32. Ozols RF: Systemic therapy for ovarian cancer: current status and new treatments. Semin Oncol 33: S3-S11, 2006.

33. Tian C, Ambrosone CB, Darcy KM, et al: Common variants in $\mathrm{ABCB} 1, \mathrm{ABCC} 2$ and $\mathrm{ABCG} 2$ genes and clinical outcomes among women with advanced stage ovarian cancer treated with platinum and taxane-based chemotherapy: a Gynecologic Oncology Group study. Gynecol Oncol 124: 575-581, 2012.

34. Catlett-Falcone R, Landowski TH, Oshiro MM, et al: Constitutive activation of Stat 3 signaling confers resistance to apoptosis in human U266 myeloma cells. Immunity 10: 105-115, 1999.

35. Pu YS, Hour TC, Chuang SE, Cheng AL, Lai MK and Kuo ML: Interleukin-6 is responsible for drug resistance and anti-apoptotic effects in prostatic cancer cells. Prostate 60: 120-129, 2004.

36. Conze D, Weiss L, Regen PS, et al: Autocrine production of interleukin 6 causes multidrug resistance in breast cancer cells. Cancer Res 61: 8851-8858, 2001.

37. Wimmel A, Glitz D, Kraus A, Roeder J and Schuermann M: Axl receptor tyrosine kinase expression in human lung cancer cell lines correlates with cellular adhesion. Eur J Cancer 37: 2264-2274, 2001.

38. Linger RM, Keating AK, Earp HS and Graham DK: Taking aim at Mer and Axl receptor tyrosine kinases as novel therapeutic targets in solid tumors. Expert Opin Ther Targets 14: 1073-1090, 2010.

39. Ye X, Li Y, Stawicki S, et al: An anti-Axl monoclonal antibody attenuates xenograft tumor growth and enhances the effect of multiple anticancer therapies. Oncogene 29: 5254-5264, 2010.

40. Birt DF, Walker B, Tibbels MG and Bresnick E: Anti-mutagenesis and anti-promotion by apigenin, robinetin and indole-3-carbinol. Carcinogenesis 7: 959-963, 1986. 